\title{
THE GALACTIC NUMBER OF WOLF-RAYET STARS PRODUCED VIA CLOSE BINARY EVOLUTION
}

\author{
E.J.A. MEURS \\ Dublin Institute for Advanced Studies, School of Cosmic Physics, Ireland \\ and \\ E.P.J. VAN DEN HEUVEL \\ Sterrenkundig Instituut Anton Pannekoek, University of Amsterdam, The Netherlands
}

\begin{abstract}
Wolf-Rayet stars may have evolved from massive close binary systems, as a result of the mass transfer processes in such systems. A substantial fraction of all WR stars known is, indeed, found in close binaries, with massive early-type companions. After the outer layers of the initial primary have been removed during Roche Lobe Over-Flow, the remaining stellar core may be a helium-burning helium star, classified observationally as a WR star. The fraction of such evolved binaries among main-sequence stars can be computed, following recipes from stellar- and galactic evolution and assuming steady, standard-IMF star formation over the last $\sim 10^{9} \mathrm{yr}$ in our Galaxy. Combining these results with the stellar PMF yields the total galactic number of such objects, or alternatively the number expected within, e.g., $3 \mathrm{kpc}$ from the Sun. Comparison with observed numbers shows that the close binary produced WR stars should constitute a significant fraction of all WR stars, the exact fraction being dependent on the lower mass limit for WR star production (and also the duration of WR characteristics in the helium star's lifetime). The mass transfer processes in close binary systems probably allow this limit to be lower than in the case of a single WR star. Another favourable comparison regards the computed and observed numbers of persistent strong, massive X-ray binaries. Finally, second-phase WR stars are likely to comprise $5-10 \%$ of the number of normal, first-stage WR binaries.
\end{abstract}

Key words: stars: Wolf-Rayet - binaries - evolution

\section{WR stages in close binary evolution}

There are two ways in which close binaries may produce Wolf-Rayet systems. In close binaries that evolve according to case B (Kippenhahn \& Weigert 1967) RLOF occurs after the end of core-hydrogen burning and before the onset of core-helium burning. The original primary star is stripped of its outer layers and only the helium core is left over, which may appear as a WR star if heavier than about 6-8 $\mathrm{M}_{\odot}$. The removal of the outer hydrogen layers is likely to proceed more easily in a close binary than for a single star and may thus require a lower original mass in order to produce a WR star (20-25 $\mathrm{M}_{\odot}$ vs. $\left.30-40 \mathrm{M}_{\odot}\right)$.

After a common-envelope phase in the later evolution of a close binary (during which large mass loss is expected), a detached very tight binary may remain: the He core of the massive star (the original secondary) together with a compact companion. For masses greater than 6-8 $\mathrm{M}_{\odot}$ the former appears again as a WR star (second WR stage). These second WR stage 
systems require again a starting mass of $20-25 \mathrm{M}_{\odot}$ - but now after the first mass-transfer episode. It is expected, therefore, that only 5-10\% of the first WR stage systems produce a second stage WR star.

\section{Observed and predicted numbers of WR stars}

Combined with recipes from Galactic evolution of stellar populations, the number of WR stars resulting from the above evolutionary processes can be predicted (for details, see Meurs \& van den Heuvel 1989). Depending (mainly) on the fraction of transferred mass that is lost from the system and further on the adopted lower mass for the He core, the number of first stage WR stars is, roughly, between 200 and 400 systems and accordingly the number of second stage WR stars between 10 and 40 systems in the Galaxy. For the purpose of a comparison with observed numbers of WR stars, it is useful to restrict the discussion to systems within $3 \mathrm{kpc}$ distance from the sun: about 10-20 first stage and 0.5-2 second stage WR binaries.

Within $3 \mathrm{kpc}$ from the sun $61 \mathrm{WR}$ stars are listed by van der Hucht et al. (1988), of which 13 are SB2 WR systems (with normal star companions, thus first stage WRs) and can be confronted with the above predicted numbers. A more detailed comparison with the calculated numbers shows, conversely, that the likely lower mass for WR stars in binaries is $6( \pm 1) M_{\odot}$. The most likely identifications for second stage WRs are (mild) runaway, apparently 'single' (but probably spectroscopically binary) WR stars, of which some 1 to 4 are known.

These comparisons demonstrate the importance of close binary evolution in the production of a sizeable fraction of all WR stars. Two further points supporting this conclusion can be mentioned. First, the calculations of Meurs \& van den Heuvel (1989) show a similarly favourable comparison between predicted and observed numbers of persistent strong, massive X-ray binaries. Second, the occurrence of second stage WR stars recently received strong support from the likely identification of the companion star in Cyg X-3 as a WR star (van Kerkwijk et al. 1992). A potentially much greater number of these systems than the one Cyg X-3 definitely recognized so far is suggested in Tutukov \& Yungelson (1993).

\section{References}

van der Hucht, K.A., Hidayat, B., Admiranto, A.G., Supelli, K.R., Doom, C. 1988, $A \mathscr{E} A$ 199,217

van Kerkwijk, M.H., Charles, P.A., Geballe, T.R. et al. 1992, Nature 355, 703

Kippenhahn, R., Weigert, A. 1967, Zeitschrift für Astrophysik 65, 251

Meurs, E.J.A., van den Heuvel, E.P.J. 1989, $A \& A$ 226, 88

Tutukov, A.V., Yungelson, L.R. 1993, MNRAS 260, 675 